\title{
Pediatric peri-operative fractionated high-dose-rate brachytherapy for recurrent Wilms' tumor using a reconstructed Freiburg flap
}

\author{
Emily Flower, MAppScil', Kathy Ngoc Tran, BAppScil, Salman Zanjani, BAppScil, Wayne Smith, BAppScil, \\ Jonathan Karpelowsky, PhD³,4, Katie Summerhayes, MScl, Edgar Estoesta, MScl, Jennifer Chard, MBBS' \\ 'Crown Princess Mary Cancer Centre, Westmead, ${ }^{2}$ Institute of Medical Physics, University of Sydney, Camperdown, 3Pediatric Surgery Children's \\ Hospital at Westmead, Westmead, ${ }^{4}$ Discipline of Pediatrics \& Child Health, Sydney Medical School, University of Sydney, New South Wales, \\ Australia
}

\begin{abstract}
Purpose: To report peri-operative fractionated high-dose-rate (HDR) brachytherapy with a 3D customized Freiburg flap applicator to treat locally recurrent Wilms' tumor, followed by immediate hyperthermic intraperitoneal chemotherapy for a 16-year-old with a second recurrence of nephroblastoma (Wilms' tumor).

Material and methods: The tumor was excised and surgical bed was treated with fractionated HDR brachytherapy using a Freiburg flap applicator. Hyperthermic intraperitoneal chemotherapy was performed immediately after the removal of brachytherapy applicator.

Results: The Freiburg flap was successfully reconstructed to enable delivery of conformable peri-operative HDR brachytherapy. The clinical target volume (CTV) $\mathrm{D}_{90}$ was $26 \mathrm{~Gy}$ in 5 fractions.

Conclusions: Peri-operative fractionated HDR brachytherapy with a customized Freiburg flap applicator was delivered successfully across a large multi-disciplinary team.

Key words: nephroblastoma, pediatric brachytherapy, peri-operative, Wilms tumor.

\section{Purpose}

Nephroblastoma (Wilms tumor) is a rare malignancy that involves tumor developing on one or both kidneys. This cancer usually develops during early childhood. Treatment options for recurrent Wilms tumors depend on the location of relapse and the recovery of surrounding organs from previous treatments. Treatment modalities include chemotherapy, surgery, and radiation therapy. Brachytherapy has been used as internal radiation therapy to treat recurrent Wilms tumor occurring within previously irradiated area $[1,2,3,4,5,6]$.

In this case report, a treatment schedule was developed to resect the tumor followed by insertion of a customized 3D applicator constructed in theatre from a Freiburg flap. High-dose-rate (HDR) peri-operative brachytherapy was delivered ( $25 \mathrm{~Gy} / 5$ fractions) to the tumor bed with curative intent, followed by hyperthermic intraperitoneal chemotherapy (HIPEC) with cisplatin. In this paper, we focus on the brachytherapy component of the treatment.

\section{Case history}

A 16-year-old boy presented with a recurrent Wilms tumor in his pelvis. He was initially diagnosed with stage 3 Wilms tumor in 2001 at age 2 and had left nephrectomy, followed by chemotherapy and whole abdominal external beam radiotherapy (EBRT) with the dose of 10.8 Gy in 6 fractions. In 2012, at age 13, he presented with his first recurrence, which was a large mass in his central abdomen abutting on his aortic bifurcation. This mass was treated with chemotherapy, tumor resection, and EBRT. The EBRT given included whole abdominal and pelvic treatment of $15 \mathrm{~Gy}$ in 10 fractions with the remaining right kidney shielded and boost of 10.5 Gy in 7 fractions to the tumor bed.

In 2015, his second recurrence of Wilms tumor developed around the level of bifurcation of common iliac vessels, at axial level of S1 and indenting the base of his bladder. The tumor was measured approximately $7 \mathrm{~cm}$ (transverse) x $6.5 \mathrm{~cm}$ (anterior-posterior) x $6.5 \mathrm{~cm}$ (craniocaudal). On magnetic resonance imaging (MRI), positron
Address for correspondence: Emily Flower, MAppSci, Crown Princess Mary Cancer Centre Westmead, Westmead Hospital, Westmead NSW 2145, Australia, phone: +31 02 88903721,

e-mail: emily.flower@health.nsw.gov.au
Received: 24.05 .2016

Accepted: 19.08 .2016

Published: 31.10 .2016 


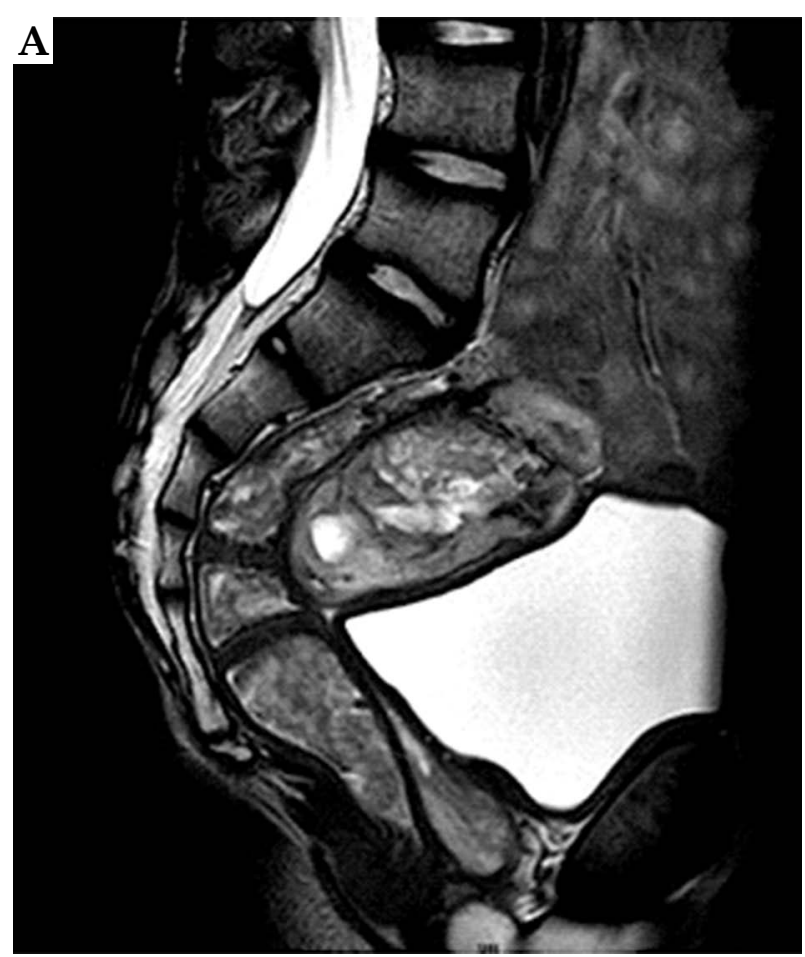

Fig. 1. The recurrent Wilms' tumor with necrotic center

emission tomography (PET), and computed tomography (CT) scans, it appeared to have necrotic center (Figure 1). This recurrence overlapped with the previous radiation treatment field. The patient was given 2 cycles of irinotecan/vincristine, followed by 2 cycles of actinomycin C/ cyclophosphamide. However, the tumor volume did not decrease significantly.

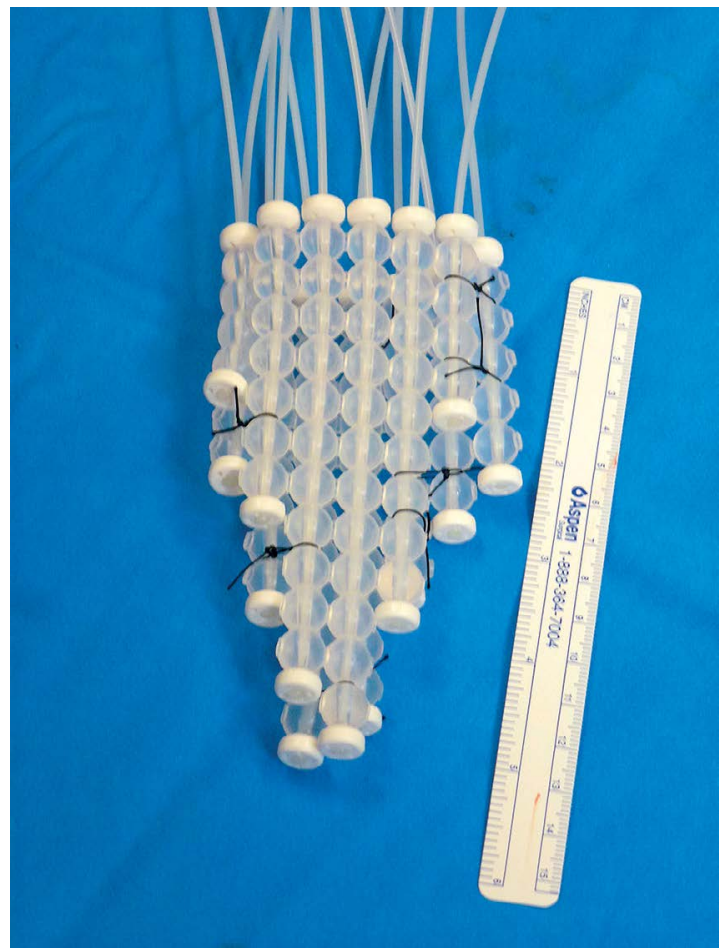

Fig. 2. The final shape of Freiburg flap applicator

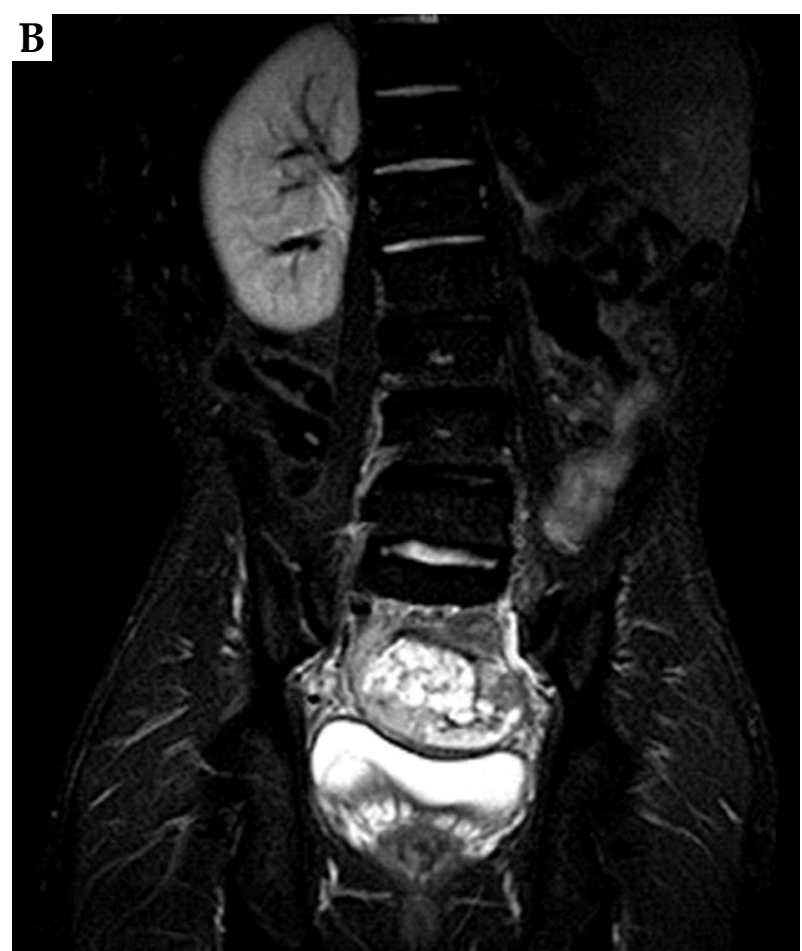

\section{Material and methods}

An applicator for HDR brachytherapy was inserted following tumor excision. At the time of excision, the right ureter (the patient's only remaining active ureter) was found to be involved by the tumor. Therefore, the decision was made to resect the right ureter and to use a temporary external drain from the right kidney.

A Freiburg flap (Nucletron, an Elekta company, Elekta $\mathrm{AB}$, Stockholm, Sweden) applicator is designed to be used for skin and surface treatment areas and intra-operative treatments. It can be implanted in the patient's body for up to 28 days. The Freiburg flap is a mesh of silicone rubber spheres with multi-planar flexibility, therefore it can be easily shaped to fit curved treatment areas. Each sphere has the separation of $5 \mathrm{~mm}$ from the central catheter to the outer edge. The flap was steam sterilized according to the manufacturer's instruction. Flexible implant tubes and plastic buttons were delivered sterile.

For this patient, the Freiburg flap applicator was trimmed down to the width of $13 \mathrm{~cm}$ and length of longest channel was $12 \mathrm{~cm}$. This enabled the 3D shape to match the surgical bed. Implant tubes were threaded through 13 channels with plastic buttons attached to both ends of every channel to stabilize the position of each tube. The brachytherapy team transformed this flap into a three-dimensional applicator by attaching the first and last channels together using 0.0 silk sutures (Figure 2).

After the Freiburg flap applicator was inserted in the surgical bed, packing was used to fill up the hollow space in the middle of the applicator to maintain its shape. Packing was inserted between applicator and intestines to push this organ at risk away from the treatment area. Two external stitches were used to keep the applicator in 


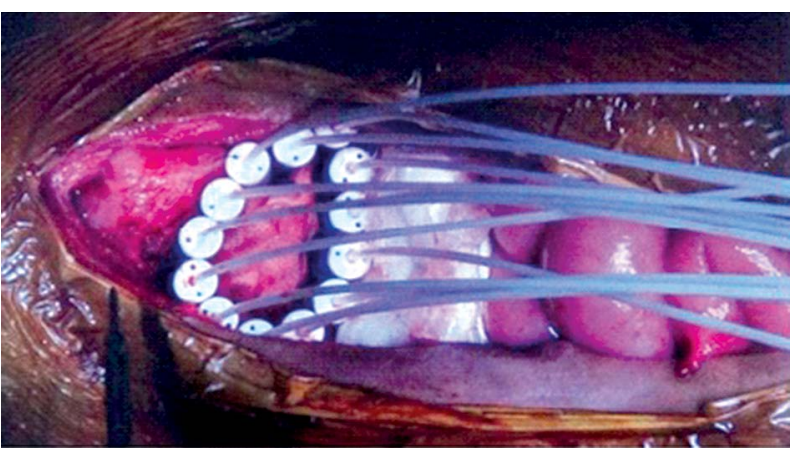

Fig. 3. Freiburg flap applicator inserted in tumor bed cavity with surgical packing to hold the applicator shape and add distance to the bowel

place. To avoid damaging and bending the implant tubes when closing the wound, the surgeon chose to use a vacuum dressing for temporary abdominal wound closure (Figures 3 and 4).

After the surgery, the patient remained under the care of the pediatric intensive care unit. Pain management was a concern; thus, patient was kept under light sedation for the duration of the treatment. A planning CT scan for HDR brachytherapy treatment was done with marker wires inserted into the implant tubes using a GE Lightspeed CT scanner (GE, Milwaukee USA) with $1.25 \mathrm{~mm}$ slice thickness. The bladder was filled with $100 \mathrm{ml}$ of water for the CT, planning and treatment for the purpose of pushing part of the bladder away from treatment area.

The prescription for this HDR brachytherapy treatment was decided to be higher than previous EBRT prescriptions. A prescribed dose of $25 \mathrm{~Gy}$ in 5 fractions was chosen to be delivered over 2 and half days, with two fractions per day and minimum of 6-hour interval between each fraction.

Treatment was planned with Varian BrachyVision (Varian Medical Systems, California, USA) treatment planning system. A margin of $0.5 \mathrm{~cm}$ surrounding the applicator and high risk area was treated as clinical target volume. An additional high risk tumor bed area was defined as the tissue locating superiorly and posteriorly to the bladder where the tumor initially located. Organs at risks included bladder, rectum, sigmoid, and bowel (Figure 5).

Treatment was delivered using the Varian Varisource iX Afterloader (Varian Medical Systems, California, USA). The applicator was connected to the afterloader using $6 \mathrm{~F}$ catheter transfer guide tubes. Treatment quality assurance included checking channel length for all 13 channels, and completing pre-treatment setup and radiation safety checklist before each fraction. Bi-daily treatment was delivered on day 3 and day 4 . Verification CT scans were performed each morning to confirm that the applicator's position was matching with the one in original plan. As the applicator was stitched into the patient's pelvis, a specific plan for responding to loss of control of the radiation source was developed. Literature reports were consulted in developing staff safety responsibilities [7]. Staff from radiation oncology, surgery, and intensive

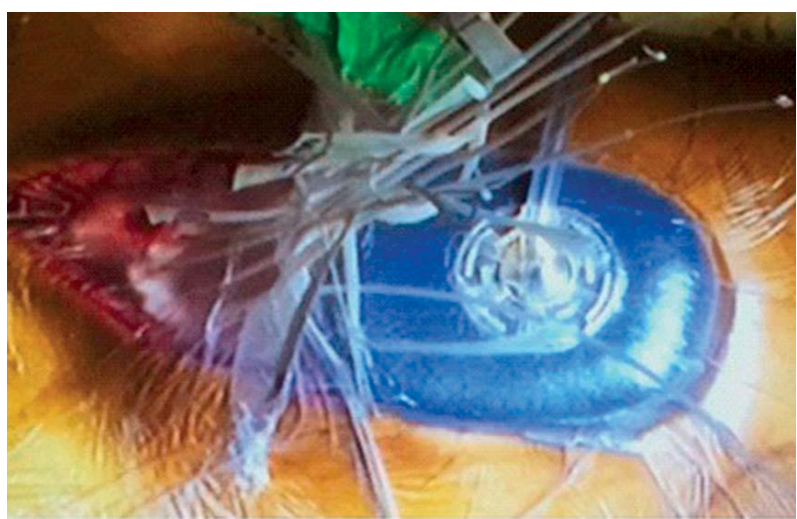

Fig. 4. Vacuum dressing was performed

care unit all had specified roles for responding to an incident should it occur, in accordance with the local regulations [8]. This formed part of the treatment protocol.

The Freiburg flap applicator was removed, followed by immediate Cisplatin HIPEC treatment.

\section{Results}

Table 1 shows the doses delivered to the clinical target volume (CTV) and organs at risk. The brachytherapy doses were converted to equivalent dose at $2 \mathrm{~Gy}\left(\mathrm{EQD}_{2}\right)$ doses using the linear quadratic model. An $\alpha / \beta$ ratio of 3 was used for organs at risk (OAR) and 10 the CTV. Total doses, including doses from previous external beam radiotherapy were also calculated. Doses received by all organs at risk were under tolerance, taking into account the doses from previous treatments. No radiobiology repair was included in these doses, but with repair the OAR total doses would be even lower.

Treatment was delivered successfully without medical or radiation emergency. Verification scans showed the applicator position was stable in relation to movement and swelling of surrounding tissues and high risk area. Re-planning was not necessary, therefore the original plan was used to treat all fractions.

\section{Discussion and conclusion}

This was a large multi-disciplinary treatment, including staff from multiple departments across two different hospitals. There were extensive collaborative discussions at national and international levels prior to treatment. A similar multi-disciplinary approach in children has been successfully performed previously [9].

Brachytherapy was chosen over repeat EBRT due to overlap with previous treatment and OAR being close to limits. The steep dose gradients and high conformality offered by brachytherapy made it the modality of choice. The Freiburg flap enabled a customized HDR brachytherapy applicator that was in direct contact with the surrounding tumor bed to be developed. Organs at risk doses were acceptable with a higher prescribed dose than previous EBRT treatments.

A twelve-month follow-up scan was performed and no evidence of local recurrence was evident. Extended 

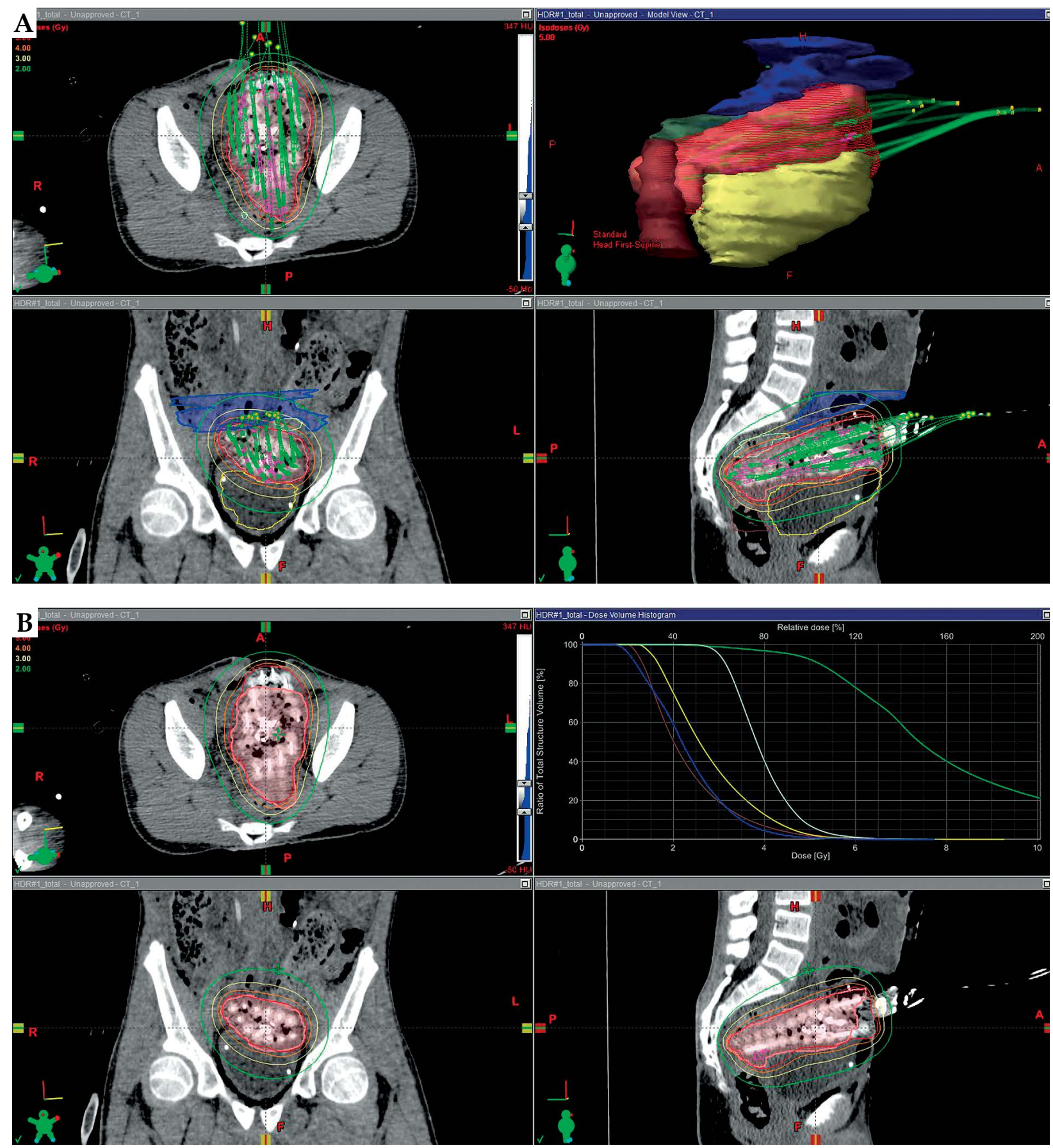

Fig. 5. Isodose distribution and dose-volume histogram

Table 1. Doses to clinical target volume (CTV)/organs at risk

Dose per fraction Total brachytherapy dose (Gy) Total EQD 2 dose (brachytherapy and previous EBRT) (Gy)

\begin{tabular}{llll}
\hline$C T V D_{90}$ & 5.2 & 32.9 & $N / A$ \\
\hline Bladder $D_{2 c c}$ & 5.2 & 42.6 & 66.5 \\
\hline Rectum $D_{2 c c}$ & 4.1 & 29.1 & 53.0 \\
\hline Sigmoid $D_{2 c c}$ & 4.2 & 30.2 & 54.1 \\
\hline Bowel $D_{2 c c}$ & 4.5 & 33.8 & 67.2
\end{tabular}

$E Q D_{2}$ - equivalent dose at $2 G y, E B R T$ - external beam radiation therapy, $D_{90}$ - the minimum dose received by $90 \%$ of the prostate volume, $D_{2 c c}-$ minimum dose to the most exposed $2 \mathrm{~cm}^{3}$ 
follow-up will be required to determine the success of the treatment and long term toxicity. The toxicity from this program of treatment included acute renal impairment (anticipated from HIPEC), weight loss, and superior mesenteric artery syndrome. An 18-day admission to the intensive care unit and further 9-week hospital admission was needed. His weight steadily improved with nasojejunal feeding and creatinine stabilized to pre-treatment levels. No direct toxicity could be ascribed to the brachytherapy component of treatment. The dose to organs at risk was below tolerance thresholds, which were conservative in not including any element of radiobiological repair. Late side effects from brachytherapy are also anticipated to be unlikely for this reason.

\section{Disclosure}

Authors report no conflict of interest.

\section{References}

1. Gandhi S, Meech SJ, Puthawala MA et al. Combined computed tomography-guided radiofrequency ablation and brachytherapy in a child with multiple recurrences of Wilms' tumor. J Pediatr Hematol Oncol 2005; 27: 377-379.

2. McManus MC, Silliman C, Koyle MA. Combined endoscopic resection and brachytherapy for recurrent intrapelvic Wilms tumor. J Urol 2002; 167: 2540.

3. Thoms WW Jr, Goldwein JW, D' Angio GD. A technique for the use of afterloading 137Cs brachytherapy in renal-sparing irradiation of bilateral Wilms tumor. Int J Radiat Oncol Biol Phys 1997; 39: 1121-1124.

4. Cherlow JM, Syed AM, Puthawala A et al. Endocurietherapy in pediatric oncology. Am J Pediatr Hematol Oncol 1990; 12: 155-159.

5. Cooper CS, Jaffe WI, Huff DS et al. The role of renal salvage procedures for bilateral Wilms tumor: a 15-year review. J Urol 2000; 163: 265-268.

6. Duckett $\mathrm{CP}, \mathrm{Zderic} \mathrm{S}$, Goldwein J et al. Brachytherapy for residual intra-renal Wilms' tumor. Med Pediatr Oncol 1997; 28: 316-320.

7. Rodriguez-Paz JM, Mark LJ, Herzer KR et al. A novel process for introducing a new intraoperative program: a multidisciplinary paradigm for mitigating hazards and improving patient safety. Anesth Analg 2009; 108: 202-210.

8. Safety Guide for Radiation Protection in Radiotherapy. ARPANSA 2008; http://www.arpansa.gov.au/publications/codes/ rps14_3.cfm.

9. Blank L, Koedooder K, Pieters B et al. The AMORE protocol for advanced-stage and recurrent nonorbital rhabdomyosarcoma in the head-and-neck region of children: a radiation oncology view. Int J Radiat Oncol Biol Phys 2009; 74: 1555-1562. 